\title{
Contralateral delay activity tracks the influence of Gestalt grouping principles on active visual working memory representations
}

\author{
Dwight J. Peterson $^{1}$ • Filiz Gözenman ${ }^{2}$ - Hector Arciniega ${ }^{2} \cdot$ Marian E. Berryhill $^{2}$
}

Published online: 28 May 2015

(C) The Psychonomic Society, Inc. 2015

\begin{abstract}
Recent studies have demonstrated that factors influencing perception, such as Gestalt grouping cues, can influence the storage of information in visual working memory (VWM). In some cases, stationary cues, such as stimulus similarity, lead to superior VWM performance. However, the neural correlates underlying these benefits to VWM performance remain unclear. One neural index, the contralateral delay activity (CDA), is an event-related potential that shows increased amplitude according to the number of items held in VWM and asymptotes at an individual's VWM capacity limit. Here, we applied the CDA to determine whether previously reported behavioral benefits supplied by similarity, proximity, and uniform connectedness were reflected as a neural savings such that the CDA amplitude was reduced when these cues were present. We implemented VWM change-detection tasks with arrays including similarity and proximity (Experiment 1); uniform connectedness (Experiments 2a and 2b); and similarity/ proximity and uniform connectedness (Experiment 3 ). The results indicated that when there was a behavioral benefit to VWM, this was echoed by a reduction in CDA amplitude, which suggests more efficient processing. However, not all perceptual grouping cues provided a VWM benefit in the same measure (e.g., accuracy) or of the same magnitude. We also
\end{abstract}

Dwight J. Peterson

petersondj@missouri.edu

$\triangle$ Marian E. Berryhill

mberryhill@unr.edu

1 Memory and Cognitive Aging Laboratory, Department of Psychological Sciences, University of Missouri-Columbia, 9J McAlester Hall, Columbia, MO 65211, USA

2 Program in Cognitive and Brain Sciences, Department of Psychology, University of Nevada, Reno, NV 89557, USA found unexpected interactions between cues. We observed a mixed bag of effects, suggesting that these powerful perceptual grouping benefits are not as predictable in VWM. The current findings indicate that when grouping cues produce behavioral benefits, there is a parallel reduction in the neural resources required to maintain grouped items within VWM.

Keywords Visual working memory · Gestalt principles · Perceptual organization · Contralateral delay activity

\section{Extending VWM capacity limitations by Gestalt principles of grouping}

Visual working memory (VWM) underlies the temporary storage and active manipulation of visual information. The amount of information from our visual world that can be stored in VWM, however, is extremely limited (Baddeley, 2010; Hollingworth, 2006; Simons \& Levin, 1997; Simons \& Rensink, 2005). Behavioral estimates of capacity suggest an upper limit of $\sim 3-4$ items, though this number is debated (Bays \& Husain, 2008; Cowan, 2001; Fukuda, Awh, \& Vogel, 2010; Luck \& Vogel, 2013). Converging evidence from electroencephalography (EEG), event-related potentials (ERP), and functional magnetic resonance imaging (fMRI) suggests that VWM capacity is constrained by the availability of a finite amount of neurobiological resources required to store information (Anderson, Vogel, \& Awh, 2011; Todd \& Marois, 2004; Vogel \& Machizawa, 2004; Xu \& Chun, 2006).

These VWM capacity limitations have prompted researchers to determine their basis and to identify ways of maximizing VWM. One area of interest has been to test whether performance benefits observed in perception extend to VWM. Specifically, Gestalt principles of grouping (e.g., similarity, proximity, common fate, continuity) and related grouping cues 
(e.g., uniform connectedness) facilitate perceptual performance (Palmer \& Rock, 1994; Wertheimer, 1950; for a recent review, see Wagemans et al., 2012) by preattentively (Duncan, 1984; Duncan \& Humphreys, 1989; Kahneman \& Treisman, 1984; Moore \& Egeth, 1997; Neisser, 1967; but see Mack \& Rock, 1998; Mack, Tang, Tuma, Kahn, \& Rock, 1992) parsing visual scenes into component objects (Duncan, 1984; Kahneman \& Henik, 1977; Neisser, 1967). Given this perceptual benefit, it is logical to anticipate that these cues would preferentially bias grouped items' entry into VWM (Woodman, Vecera, \& Luck, 2003) and improve VWM performance. In describing the current suite of experiments, we borrow terms from the perceptual organization literature when referring to specific "grouping cues" and their impact on VWM processes when referring to "grouping-related benefits."

Indeed, the VWM literature contains reports of improved VWM performance when grouping cues are available (strong collinearity: Anderson, Vogel, \& Awh, 2013; similarity: Gao et al., 2011; Lin \& Luck, 2009; Morey, Cong, Zheng, Price, \& Morey, 2015; Quinlan \& Cohen, 2012; similarity and proximity: Brady \& Tenenbaum, 2013; Peterson \& Berryhill, 2013; Shen, Yu, Xu, \& Gao, 2013; amodal completion: Walker \& Davies, 2003; connectedness and proximity: Woodman et al., 2003; Xu, 2006; common region: Xu \& Chun, 2007; depth cues: Kristjánsson, 2006; and contextual grouping: Jiang, Chun, \& Olson, 2004). Grouping may also add noise to estimates of VWM capacity because some paradigms introduce incidental grouping cues (e.g., similarity, proximity) by choosing stimuli with replacement (e.g., Luck \& Vogel, 1997). Incidental grouping provides similarity and proximity cues and improves VWM performance compared to arrays without grouping cues (Brady \& Tenenbaum, 2013). Furthermore, grouping cues may not provide equal benefits and may not reflect the same underlying mechanism(s).

The underlying mechanism(s) are unknown. One proposed explanation is that grouping reduces the neural requirements to maintain items in VWM, potentially by providing a redundant signal benefit or by taking advantage of object-based attention. VWM delay-related activity is lower for stimulus arrays containing grouped items in posterior regions measured using ERP (Gao et al., 2011) and fMRI (e.g., intraparietal sulcus, connectedness: Xu, 2008; common region: Xu \& Chun, 2007; but see Berryhill, Peterson, Jones, \& Stephens, 2014). One way of monitoring VWM contents is by measuring the contralateral delay activity (CDA). The CDA is an ERP waveform derived by subtracting the activity associated with ipsilateral hemifield stimulation from the activity associated with contralateral hemifield stimulation. It is calculated from posterior electrode sites, emerges around 400 milliseconds poststimulus onset, is sustained during the VWM maintenance period, increases in amplitude with VWM load, and reaches asymptote at an individual's capacity limit (Vogel \& Machizawa, 2004). Source localization suggests it arises from the area in and around the superior parietal lobe (Gao et al., 2011). Grouping also reduces CDA amplitudes. For instance, when distinct stimuli (e.g., wrench heads) were oriented to form collinear groups, the CDA amplitude was significantly smaller than when the same stimuli were rotated (Anderson et al., 2013). In addition, the CDA amplitude is equal when storing one item of one color (e.g., one blue square) or four identical items (e.g., four blue squares; Gao et al., 2011).

As noted, the literature contains many reports of VWM benefits from established perceptual grouping cues. However, these grouping benefits do not appear to be of equal robustness in VWM as in perception, as evidenced by failures to replicate VWM benefits (e.g., common region: Berryhill et al., 2014; for a successful replication in similarity, see Peterson \& Berryhill, 2013). Second, VWM grouping benefits are likely not produced by a single neural mechanism. Here, we use the CDA to serve as a measure of the neural resources devoted to VWM that can clarify the mechanism(s) associated with any observed grouping benefits. In other words, leveraging the benefits of grouping cues may reveal where cognitive savings can be made to enhance VWM.

Experiment 1 examined the influences of grouping via similarity of color and spatial proximity. One recent study found that similarity alone did not modulate CDA amplitude (Shen et al., 2013). However, spatial proximity was not controlled for, as the locations of similar items were randomized. Previously, we found that proximity aids in developing a VWM benefit from similarity (Peterson \& Berryhill, 2013; but see Morey et al., 2015). In Experiments $2 \mathrm{a}$ and 2b, we turned to a second well-established grouping cue, uniform connectedness, because it also has been reported to provide VWM benefits (Woodman et al., 2003; Xu, 2006; but see Berryhill et al., 2014), which may rely on regions within the posterior parietal cortex (e.g., Xu, 2008). Finally, in Experiment 3, we measured the consequences of combining similarity and uniform connectedness in the same study to examine the relative and potential superadditive benefits to VWM. We predicted that if behavioral benefits to VWM emerged, they would be accompanied by a reduction in CDA amplitude for grouped compared to ungrouped arrays of the same set size if they reflected the same underlying neural mechanism, and a disconnect between behavioral and CDA patterns if there were different underlying neural mechanisms.

\section{Experiment 1: Similarity and proximity}

\section{Method}

\section{Participants}

Twenty-two undergraduate students from the University of Nevada, Reno, participated in Experiment 1 (14 female, mean 
age $=22.2$ years). To enter group-level analyses, we imposed a minimum average behavioral performance criterion of $70 \%$ response accuracy. As such, seven participants were excluded. All participants were right-handed, neurologically intact, and had normal or corrected-to-normal color vision. The Institutional Review Board at the University of Nevada, Reno, approved all experimental protocols.

\section{Apparatus}

The experimental task and stimuli were generated and presented with MATLAB (Mathworks, Natick, MA) using the Psychophysics Toolbox 3.0 extension (Brainard, 1997; Pelli, 1997). Stimuli were displayed on a 19-inch NEC MultiSync E1100 CRT monitor (refresh rate of $75 \mathrm{~Hz}$ at a resolution of $1024 \times 768)$ via a Mac mini $2.5 \mathrm{GHz}$ dual-core Intel Core i5.

\section{Stimuli and procedure}

Colored squares $\left(0.7 \times 0.7^{\circ}\right)$ were randomly chosen from a set of seven colors (cyan, white, red, blue, yellow, green, magenta). Stimuli were presented symmetrically at three possible locations on each side of fixation $\left(5.2^{\circ}\right)$; see Fig. 1a. There were four conditions. In the two ungrouped item condition (2UG), two differently colored squares were presented in two of the three locations. In the three ungrouped item condition (3UG), three differently colored squares were presented. For the grouped conditions, two of the three squares shared the same color and were considered three items grouped via similarity and strong proximity (3-SSP) when the matching squares were neighbors, and three items grouped via similarity and weak proximity (3-SWP), when the matching squares were separated by a nonmatching square.

Trials began with the presentation of a black fixation cross $\left(0.4^{\circ} \times 0.4^{\circ}, 300 \mathrm{~ms}\right)$, followed by the presentation of a left or right black arrow $\left(2.1^{\circ} \times 0.4^{\circ}, 200 \mathrm{~ms}\right)$ above fixation cueing the side of the array to covertly attend during encoding. After a variable delay (300-400 ms) the VWM array was presented $(100 \mathrm{~ms})$. Stimuli were presented in two rectangular areas subtending $7.1^{\circ} \times 12.2^{\circ}$ of visual angle centered $4.6^{\circ}$ to the left or right of the fixation cross on a uniform medium gray background. Participants viewed the stimuli from a distance of $57 \mathrm{~cm}$. After a delay-period $(900 \mathrm{~ms})$, a single probe stimulus appeared ( $3 \mathrm{~s})$. A single probe was used to keep the current task design consistent with previous research examining the impact of grouping cues on the CDA (Gao et al., 2011; Shen et al., 2013) and to prevent participants from making their decision based on the initial spatial configuration between items (Jiang, Olson, \& Chun, 2000). Participants indicated whether the color of the probed item matched ("o" key, $50 \%$ ) or mismatched (" $n$ " key) the original stimulus item. If no response was registered, the trial was considered incorrect and screen instructions appeared, stating to press any key to continue to the next trial. Prior to beginning the experiment, participants completed 24 practice trials. Thirteen blocks (with the opportunity for self-paced breaks between blocks) of 48 trials per block were presented for a total of 624 trials, with 156 trials per condition. Trial types were randomly interleaved within each block. Participants were instructed to maintain fixation and to avoid voluntary eye movements. Performance measures included accuracy, reaction time and VWM capacity estimates: $\mathrm{K}=$ Set size*(Hit rate - False alarm rate); (Cowan, 2001; Pashler, 1988).

\section{Electroencephalography recordings}

The EEG was recorded at a sampling rate of $1000 \mathrm{~Hz}$ with a vertex $(\mathrm{Cz})$ reference from 256 high-impedance electrodes mounted in a HydroCel Geodesic Sensor Net amplified by a Net Amps 300 amplifier and acquired using Net Station 4.5.5 software (Electrical Geodesics Inc., Eugene, OR) running on a 2.7 GHz dual-core Apple Power Mac G5. EEG data were rereferenced off-line to the average of the left and right mastoids. Individual continuous EEG datasets were filtered using finite impulse response (FIR) filters high-passed at $0.01 \mathrm{~Hz}$ and low-passed at $30 \mathrm{~Hz}$ off-line. The EEG data corresponding to correct trials were segmented by condition using an epoch of $200 \mathrm{~ms}$ (baseline period) before stimulus onset and ending 1,000 ms after stimulus onset.

Artifact detection and rejection routines eliminated eye movements, blinks, and channel loss. The electrode sites around the eyes provided data for artifact detection and rejection routines. Segments contaminated by blinks or eye movements $\left(>1^{\circ}\right)$ were rejected prior to averaging (threshold criteria: eye movements: $20 \mu \mathrm{V}$, blinks: $150 \mu \mathrm{V}$ ). Trials were excluded if they contained residual artifacts (e.g., ocular artifact, movement artifact, amplifier saturation) exceeding $\pm 75 \mu \mathrm{V}$ from $600 \mathrm{~ms}$ prestimulus to $1,000 \mathrm{~ms}$ poststimulus onset. Bad channels (e.g., $>50 \mathrm{~K} \Omega$ electrode impedance, line noise, drift) were detected and replaced using interpolation algorithms implemented by Net Station 4.5.5. software (Electrical Geodesics Inc., Eugene, OR). Finally, the segmented EEG data for correct trials from each condition were averaged to generate ERPs for each participant and each condition. Baseline correction was performed using the baseline period.

\section{CDA analysis}

The CDA is calculated as a difference score between responses to stimuli presented to the contralateral and ipsilateral hemifields (Vogel \& Machizawa, 2004). For all experiments, average contralateral and ipsilateral waveforms for each condition were computed at posterior sites typically examined in CDA research (left hemisphere posterior sites: P7, PO7, TP7; right hemisphere posterior sites: P8, PO8, TP8). Difference waveforms for each electrode pair (P7-P8, PO7-PO8, TP7- 
Fig. 1 Experiment 1 task paradigm, stimulus configurations, and behavioral results. A. Participants viewed a fixation cross $(300 \mathrm{~ms})$ and then were given an arrow cue $(200 \mathrm{~ms})$ indicating the side of the screen to which they should covertly attend during the trial. A variable interval $(300-400 \mathrm{~ms})$ preceded the memory array $(100 \mathrm{~ms})$ containing one of the four conditions displayed above. The experimental conditions included stimulus displays including either two items (2-UG), three items (3-UG), three items grouped by similarity and weak proximity (3SWP), or three items grouped by similarity and strong proximity (3-SSP). After a delay-period ( $900 \mathrm{~ms})$, a probed item appeared that was either the same color ("old" trials) originally presented or a different color ("new" trials). Participants were given 3 seconds to respond. Note that stimuli are depicted for illustrative purposes only and do not reflect the exact dimensions and visual angles of the stimuli displayed during the actual experiment. B-C. The abscissa depicts the conditions being compared while accuracy (proportion correct) is plotted along the ordinate. B. Overall VWM performance in Experiment 1. C. VWM performance based on the two probe types for trials from both the three object similarity + weak proximity condition and the three object similarity + strong proximity, which could have been a colored square that was grouped or ungrouped during stimulus presentation. In panels B and C, asterisks symbolize an observed significant difference between various conditions; $\alpha=p<.05$. Error bars represent the standard error of the mean in each condition.

TP8) were created by subtracting the average activity recorded from ipsilateral sites from the average activity recorded from contralateral sites. These data were then averaged across electrode pairs. The time window used to measure the CDA was 400-1,000 milliseconds poststimulus onset. Because the literature does not have a standard convention, we report all electrode pairs separately to facilitate comparison with other findings.

\section{Results}

\section{Behavioral results}

To examine whether the presence of similarity and proximity improved VWM performance, we analyzed several behavioral measures (e.g., accuracy, estimated capacity (K), and reaction time). As indicated by a repeated-measures ANOVA including the within-subjects factor of condition (2-UG, 3-UG, 3-SWP, 3-SSP), there was a significant difference in accuracy across conditions, $\mathrm{F}(3,42)=26.67, M S E=0.022, p<.001$, $\eta_{p}{ }^{2}=0.66, \beta=0.99$. Bonferroni corrected pairwise comparisons indicated that this was driven by significantly lower performance in the 3-UG $(M=0.84)$ condition compared to all other conditions $(2-\mathrm{UG}=0.91 ; 3-\mathrm{SWP}=0.93 ; 3-\mathrm{SSP}=0.91$; all $p \mathrm{~s}<.001$ ); see Fig. 1b. These data confirmed a significant similarity benefit regardless of proximity, consistent with recent results (Morey et al., 2015).

A subsequent $2 \times 2$ repeated-measures ANOVA examined accuracy as a function of probe type (probing a grouped or ungrouped item) and strength of proximity between items (i.e., the 3-SSP and 3-SWP conditions). There was a main
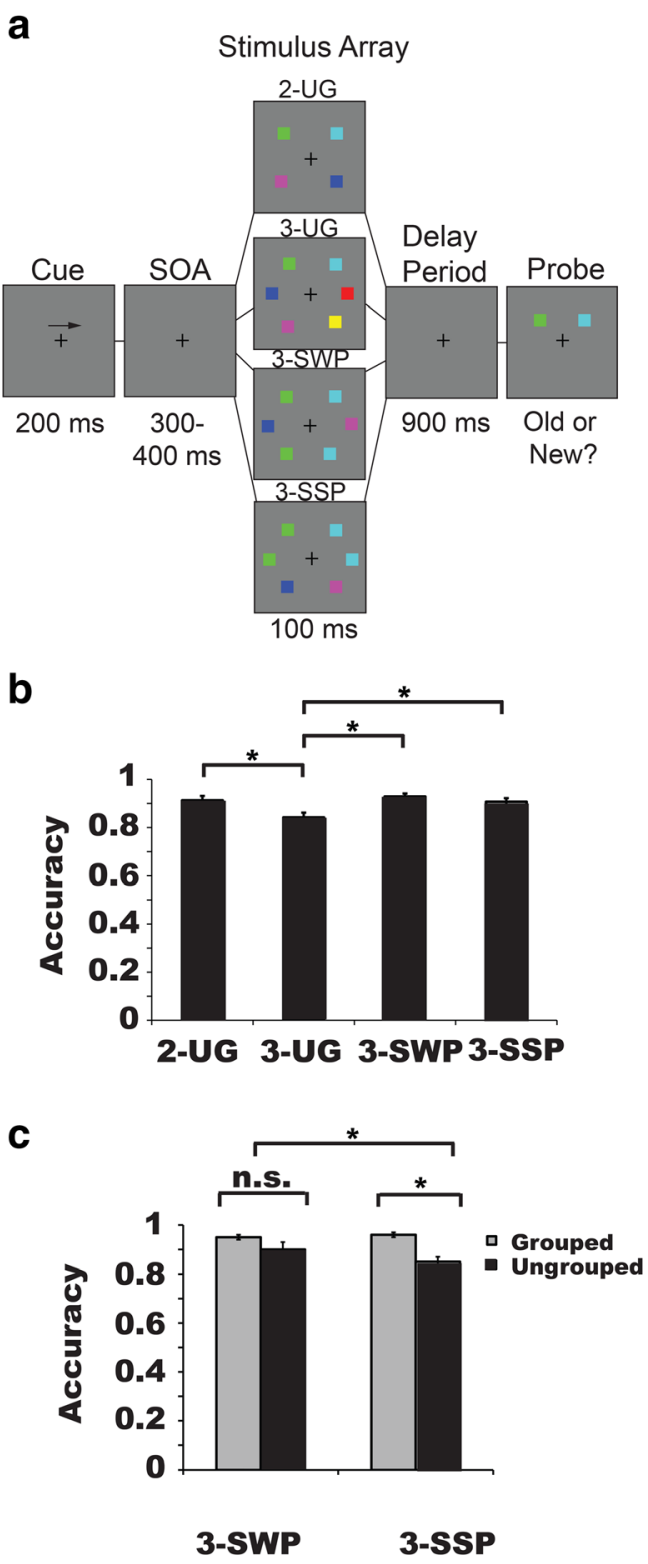

effect of probe type, $F(1,14)=18.31, M S E=0.09, p=$ $.001, \eta_{p}{ }^{2}=0.57, \beta=0.98$, corresponding with higher accuracy for trials probing a grouped item $(M=0.95)$ than an ungrouped item $(M=0.87)$; see Fig. 1c. Additionally, there was a main effect of proximity, $F(1,14)=5.44, M S E=0.008, p=$ $.04, \eta_{p}{ }^{2}=0.28, \beta=0.58$, such that accuracy for the 3-SWP condition was higher $(M=0.93)$ than in the 3 -SSP condition $(M=0.91)$. Finally, there was a significant interaction between probe type and proximity, $F(1,14)=7.35, M S E=0.02, p=$ $.02, \eta_{p}{ }^{2}=0.34, \beta=0.71$, driven by a significantly greater probe effect on 3-SSP arrays (grouped: $M=0.96$, ungrouped: $M=0.85, p<.001$ ) than the 3-SWP arrays (grouped: $M=$ 0.95 , ungrouped: $\mathrm{M}=0.90, \mathrm{p}=.09$ ); see Fig. $1 \mathrm{c}$.

The accuracy analyses indicated that VWM performance benefited from similarity and proximity. To examine whether 
grouping increased capacity we subjected the Cowan's K values to a repeated-measures ANOVA revealed a significant difference in $\mathrm{K}$ across conditions, $\mathrm{F}(3,42)=97.54$, MSE = $2.59 \mathrm{p}<.001, \eta_{\mathrm{p}}{ }^{2}=0.87, \beta=0.99$. The same differences observed in the accuracy data remained significant, for example, the increase from the $2-\mathrm{UG}$ and all of the 3-item conditions (all ps $<.001$ ). In addition, there were significant differences between the 3-UG and both grouped conditions, 3-SWP and the 3-SSP, (both ps $<.001$ ), but not between the two grouped conditions (3-SWP, 3-SSP: $\mathrm{p}=.13$ ). Higher K values were associated with grouped arrays, regardless of proximity. Finally, average reaction times for each condition were consistent and showed no grouping benefit, $\mathrm{F}(3,42)=2.23, \mathrm{p}=$ .10; $2-\mathrm{UG}=1,103 \mathrm{~ms} ; 3-\mathrm{UG}=1,159 \mathrm{~ms} ; 3-\mathrm{SWP}=1,113 \mathrm{~ms}$; $3-\mathrm{SSP}=1,142 \mathrm{~ms}$.

\section{Electrophysiological results}

The CDA amplitudes followed the behavioral pattern. We focused on the electrode pair TP7/TP8, which produced the largest effect and an increase in CDA amplitude from two to three items; however, the pattern of results is similar at two other electrode pairs of interest (e.g., P7/P8, PO7/PO8; see Table 1). A repeated-measures ANOVA comparing the factor of experimental condition (2-UG, 3-UG, 3-SWP, 3-SSP) indicated a significant main effect in CDA amplitude across experimental conditions, $\mathrm{F}(3,42)=6.30, \mathrm{MSE}=14.31, \mathrm{p}=$ $.005, \eta_{\mathrm{p}}{ }^{2}=0.31, \beta=0.95$, showing the expected increase in amplitude between the 2-UG and 3-UG conditions (2-UG = $0.25 \mu \mathrm{V}, 3-\mathrm{UG}=-2.28 \mu \mathrm{V}, \mathrm{p}=.006)$. Importantly, there was a significant increase in CDA amplitude from the 3-SSP to the 3 -UG conditions ( $3-\mathrm{SSP}=-0.78 \mu \mathrm{V}, \mathrm{p}=.02$ ) and a borderline increase in CDA amplitude from the 3-SWP to the 3-UG conditions (3-SWP $=-0.17 \mu \mathrm{V}, p=.07)$. Importantly, there was no difference in the amplitude of the CDA between the 2-
UG condition and either the 3-SSP or 3-SWP conditions ( $p \mathrm{~s}=$ 1); see Fig. 2.

\section{Discussion}

Experiment 1 replicates and extends previous work confirming that arrays containing similarity of color cues benefit VWM (Brady \& Tenenbaum, 2013; Lin \& Luck, 2009; Morey et al., 2015; Peterson \& Berryhill, 2013). Behaviorally, participants performed better when storing or reporting on grouped VWM arrays than ungrouped arrays of the same set size. Importantly, the behavioral benefit to VWM was echoed in the neural data by a selective reduction in the CDA amplitude during the storage of grouped arrays. Given that the amplitude of the CDA tracks the number of distinct item identities in VWM (Gao et al., 2011; Vogel \& Machizawa, 2004), the data suggest that grouped items were integrated into a single representation. Furthermore, the CDA amplitude for each grouped condition was similar to the CDA corresponding to two separate objects (2-UG condition) but not three separate objects (3-UG condition). To test the consistency of these benefits both behaviorally and mechanistically, we next investigated uniform connectedness because of previously reported benefits to VWM.

\section{Experiment 2a: Uniform connectedness}

There are several reports of a significant benefit to VWM from uniform connectedness during change detection VWM tasks (Woodman et al., 2003; Xu, 2006). However, in both cases it was clear that the connectedness benefit emerged under carefully titrated difficulty levels (e.g., only with certain set sizes). Using fMRI, Xu and Chun (2007) linked behavioral data with activation in intraparietal regions corresponding to the

Table 1 Electrophysiological results per electrode pair from Experiment 1

\begin{tabular}{|c|c|c|c|}
\hline Electrode pair & Mean amplitude per condition $(\mu \mathrm{V})$ & Analysis of variance ( $F$ test) & Notable pairwise comparisons $(p)$ \\
\hline $\mathrm{P} 7 / \mathrm{P} 8$ & $\begin{array}{l}\text { 2-UG: }-0.54 \\
\text { 3-UG: }-1.71 \\
\text { 3-SWP: }-0.30 \\
\text { 3-SSP: }-1.05\end{array}$ & $\begin{array}{l}F(3,42)=5.01 \\
p=.007, \eta_{p}^{2}=0.26, \beta=0.89\end{array}$ & $\begin{array}{l}\text { 2-UG, 3-UG(.05)* } \\
\text { 3-UG, 3-SWP(.05)* }\end{array}$ \\
\hline PO7/PO8 & $\begin{array}{l}\text { 2-UG: }-1.09 \\
\text { 3-UG: }-1.90 \\
\text { 3-SWP: }-0.25 \\
\text { 3-SSP: }-0.21\end{array}$ & $\begin{array}{l}F(3,42)=2.74 \\
p=.09, \eta_{p}^{2}=0.16, \beta=0.62\end{array}$ & \\
\hline TP7/TP8 & $\begin{array}{l}\text { 2-UG: }-0.25 \\
\text { 3-UG: }-2.28 \\
\text { 3-SWP: }-0.17 \\
\text { 3-SSP: }-0.78\end{array}$ & $\begin{array}{l}F(3,42)=6.30 \\
p=.005, \eta_{p}^{2}=0.31, \beta=0.95\end{array}$ & $\begin{array}{l}\text { 2-UG, 3-UG(.006)* } \\
\text { 3-UG, 3-SWP(.07) } \\
\text { 3-UG, 3-SSP(.02)* }\end{array}$ \\
\hline
\end{tabular}

Note. Electrophysiological results from Experiment 1 at each electrode pair examined. The table columns indicate, from left to right, the electrode pair, the corresponding mean CDA amplitude values per condition, the statistical results, and notable pairwise comparisons. Asterisks next to the $p$ values in parentheses indicate a significant difference (alpha level: $p<.05$ ). 


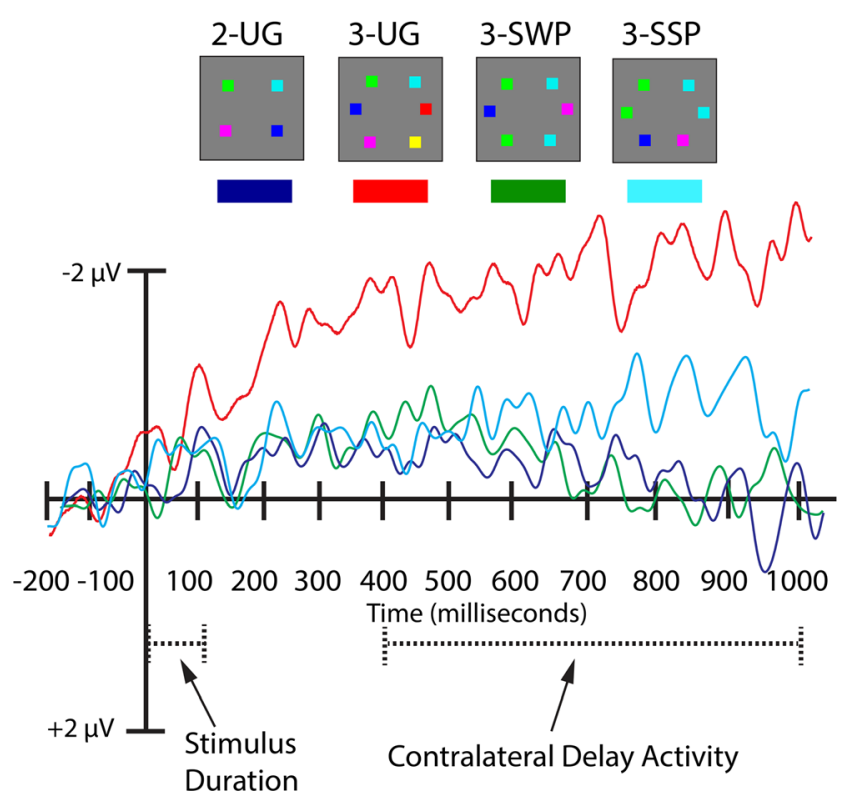

Fig. 2 Electrophysiological results for Experiment 1. The timecourse of the CDA (in $\mathrm{ms}$ ) is depicted along the abscissa. The amplitude scale (in microvolts) of the CDA for each experimental condition is indicated along the ordinate. The timecourse of the waveform depicts the prestimulus baseline period $(-200-0 \mathrm{~ms})$ occurring prior to the onset of the stimulus array for a given trial. The stimulus array was presented for $100 \mathrm{~ms}$ and was followed by a delay interval $(900 \mathrm{~ms})$. The measurement window for CDA analyses included amplitude values taken from 400$1,000 \mathrm{~ms}$ post stimulus onset. The CDA amplitudes derived from electrode pair TP7/TP8 are depicted for each experimental condition.

electrode pairs used in calculating the CDA. As such, we reasoned connectedness was a reasonable grouping mechanism to explore next. In Experiment 2a, we predicted both behavioral benefits and an accompanying reduction in the CDA. If behavioral benefits were not reflected in the CDA, we would infer a different underlying mechanism.

\section{Method}

\section{Participants}

A new group of 20 participants participated in Experiment 2a (12 female, mean age $=21.6$ years). Five participants were excluded because their behavioral performance failed to meet criterion ( $>70 \%$ correct).

\section{Stimuli and procedure}

The methods used in Experiment 2a match those used in Experiment 1 except where noted. The same stimuli and procedures were used in Experiment 2 with the following exceptions. In each condition the colored squares partially occluded a black square $\left(0.82^{\circ} \times 0.82^{\circ}\right)$ connected to a black rectangle $\left(0.65^{\circ} \times 1.8^{\circ}\right)$. These stimuli were similar to the "wrench-like" stimuli that in previous research produced a "same-object" benefit (Watson \& Kramer, 1999). In the grouped condition, two proximal black rectangles (i.e., wrench handles) formed a connected pair (3-C); see Fig. 3a. There were three conditions: 2-UG, 3-UG and 3-C. There were 204 trials per condition for a total of 612 trials.

\section{Results}

\section{Behavioral results}

The accuracy data were subjected to repeated-measures ANOVA with the factor of condition (2-UG, 3-UG, 3-C). There was a significant main effect, $F(2,28)=56.82, M S E=$ $0.051, p<.001, \eta_{p}{ }^{2}=0.80, \beta=0.99$, driven by a drop in accuracy between the 2-UG and 3-UG conditions (2-UG $=$ $0.92,3-\mathrm{UG}=0.83, p<.001)$ and between the 2-UG and 3-C conditions $(3-\mathrm{C}=0.82, p<.001)$. There was no difference between the 3-UG and 3-C conditions $(\mathrm{p}=1.0)$; see Fig. $3 \mathrm{~b}$. However, analysis of probe type (grouped, ungrouped) in the 3$\mathrm{C}$ trials revealed better performance when a grouped item was probed, grouped $=0.86$; ungrouped $=0.77 ; \mathrm{t}(14)=2.68, p=$ .02; see Fig. 3c.

Analysis of the $\mathrm{K}$ values found the same pattern. There was a main effect of condition, $\mathrm{F}(2,28)=9.20$, MSE $=$ $0.29, \mathrm{p}=.001, \eta_{\mathrm{p}}{ }^{2}=0.40, \beta=0.96$, such that there was a significant increase in $\mathrm{K}$ between the 2-UG and 3-UG conditions $(2-\mathrm{UG}=1.69,3-\mathrm{UG}=1.95, \mathrm{p}=.003)$, and the $2-\mathrm{UG}$ and $3-\mathrm{C}$ conditions $(3-\mathrm{C}=1.91, \mathrm{p}=.03)$, but not between the $3-\mathrm{UG}$ and $3-\mathrm{C}$ conditions $(\mathrm{p}=1.0)$. Finally, reaction times also differed across conditions, $F(2,28)=28.95$, $M S E=0.04, p<.001, \eta_{p}^{2}=0.67, \beta=0.99$, with significantly faster times for the 2-UG arrays $(2-\mathrm{UG}=893 \mathrm{~ms}$; both $p \mathrm{~s}=.001)$ than the 3-UG and 3-C conditions (3-UG = $975 \mathrm{~ms} ; 3-\mathrm{C}=982 \mathrm{~ms} ; p=1.0$ ).

\section{Electrophysiological results}

We focused on the data from electrode pair P7/P8 because it showed an increase in amplitude from 2-UG to 3-UG objects, however, mean amplitude values and analyses corresponding to the other two electrode pairs, which were also examined in Experiment 1 (i.e., PO7/PO8 ${ }^{1}$, TP7/TP8) are shown in Table 2. There was a significant main effect of condition, $F(2,28)=$ 3.79, $M S E=1.64, p=.04, \eta_{p}{ }^{2}=0.21, \beta=0.64$. As anticipated, the CDA amplitude was significantly smaller for the 2-UG condition $(-0.82 \mu \mathrm{V})$ than the $3-\mathrm{UG}$ condition $(-1.43 \mu \mathrm{V}, p=$ $.001)$. There was no difference between the 2-UG and the 3-C

\footnotetext{
${ }^{1}$ The lead wire corresponding to electrode site PO8 was disconnected on the geodesic sensor net (Channel 161) resulting in signal loss from this channel for one participant. This participant's data was excluded from analysis of the mean CDA amplitudes corresponding to electrode pair PO7/PO8.
} 


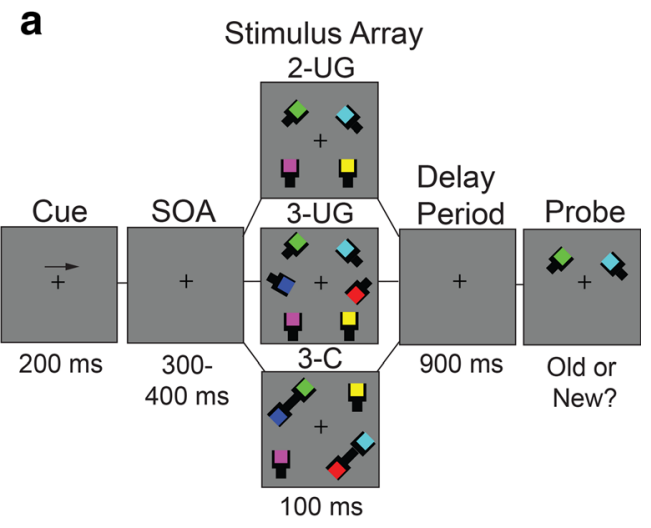

b

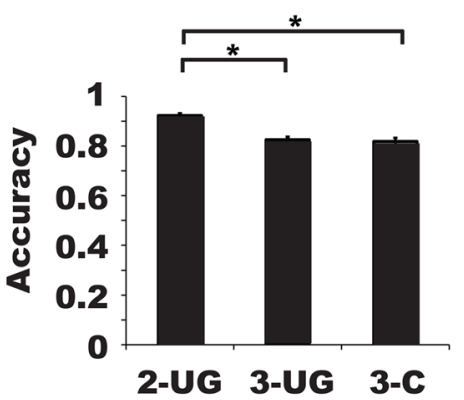

C

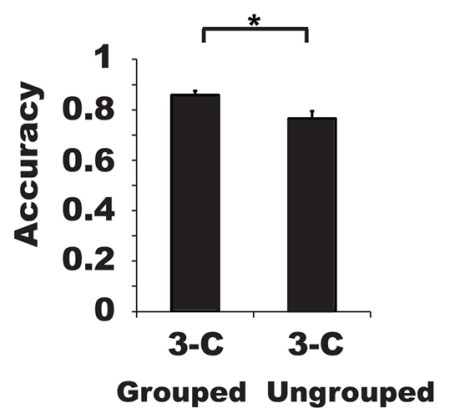

Fig. 3 Experiment 2 (a/b) task paradigm, stimulus configurations, and behavioral results for Experiment 2a. A. Participants first viewed a fixation cross $(300 \mathrm{~ms})$ and then were given an arrow cue $(200 \mathrm{~ms})$ indicating the side of the screen to which they should covertly attend during the trial. A variable interval (300-400 ms) preceded a memory array $(100 \mathrm{~ms})$ containing one of the three conditions displayed above: Two items (2-UG), three items (3-UG), or three items in which two of the items were connected (3-C). After a delay period (Experiment 2a: $900 \mathrm{~ms}$; Experiment 2b: 600, 700, 800, or $900 \mathrm{~ms}$ ), a probed item appeared that was either the same color ("old" trials) as presented originally or a different color ("new" trials). Participants were given 3 seconds to respond. Note that stimuli are depicted for illustrative purposes only and do not reflect the exact dimensions and visual angles of the stimuli displayed during the actual experiment. B. VWM performance in Experiment 2a. The abscissa depicts the stimulus conditions for Experiment $2 \mathrm{a}$. Accuracy is plotted along the ordinate by showing the proportion of correct trials in each condition. $\mathrm{C}$. The abscissa depicts the two probe types for trials from the three object connected condition, which could have been a colored square that was grouped or ungrouped during stimulus presentation. Accuracy is plotted along the ordinate by showing the proportion of correct trials for each probe type within the three-object connected condition. In panels B and C, asterisks symbolize an observed significant difference between various conditions; $\alpha=p<$ .05 . Error bars represent the standard errors of the means.
CDA amplitudes $(-1.34 \mu \mathrm{V}, p=0.22)$. No difference in CDA amplitude for the 3-UG and 3-C conditions $(p=1.0)$ was observed; see Fig. 4.

Visual inspection of the CDA suggested the possibility of distinct early and late components (for other evidence on early and late CDA effects in VWM, see Luria \& Vogel, 2011). The amplitude for the 3-C condition tracked closely with the 3-UG condition during the initial portion of the delay period but later decreased to the level of the 2-UG condition. We separately examined the early (400-600 ms) and late (800-1,000 ms) stages of the delay-period. The early delay-period showed a main effect of condition, $F 2,28)=4.51, p=.02, \eta_{p}^{2}=0.24, \beta$ $=0.72$, such that CDA amplitudes for the 2-UG $(-0.80 \mu \mathrm{V})$ condition were significantly smaller than for the 3-UG (-1.43 $\mu \mathrm{V})$ and $3-\mathrm{C}(-1.51 \mu \mathrm{V}, p \mathrm{~s}<.04)$ conditions. There was no difference between the 3-UG and 3-C conditions $(p=1.0)$. However, a significant change in this pattern emerged during the late delay period $(800-1,000 \mathrm{~ms}), F(2,28)=3.87, p=.03$, $\eta_{p}{ }^{2}=0.22, \beta=0.65$. Specifically, the CDA amplitude for 3UG $(-1.47 \mu \mathrm{V})$ remained significantly higher than for 2-UG ($0.69 \mu \mathrm{V}, p=.003)$, but there was no longer a significant difference between the 2-UG and 3-C $(-1.36 \mu \mathrm{V}, p=.23)$ conditions. As before, there was no significant difference in $\mathrm{CDA}$ amplitude between the 3-UG and 3-C conditions $(p=$ 1.0). In other words, at this late delay period the 3-C CDA amplitude was intermediate between the 2-UG and 3-UG conditions but not significantly different from either.

\section{Discussion}

We anticipated replicating an overall VWM benefit for arrays including connected stimuli, but we did not. There was a more subtle benefit. There was a VWM benefit of uniform connectedness when one of the grouped items was probed at test. Moreover, the amplitude of the CDA for the 3-C condition differed from the 3-UG condition only during the later portion of the delay period (i.e., $800-1,000 \mathrm{~ms}$ ). Intriguingly, this earlylate delay period distinction suggests that uniform connectedness benefits to VWM may depend on the length of the delay period. Specifically, while no VWM performance benefit of uniform connectedness was present during the early delay (i.e., 400-600 ms), a benefit may emerge during the later portion of the delay period (i.e., $800-1,000 \mathrm{~ms}$ ). Similar findings have been reported with respect to the neural resources required for the binding of visual features within VWM during early and late portions of the delay period (e.g., Luria \& Vogel, 2011).

\section{Experiment 2b: The influence of delay period interval on uniform connectedness}

To more carefully examine the possibility that the benefits of uniform connectedness emerge at later portions of the VWM 
Table 2 Electrophysiological Results per Electrode Pair from Experiment 2

\begin{tabular}{llll}
\hline Electrode pair & Mean amplitude per condition $(\mu \mathrm{V})$ & Analysis of variance $(F$ test $)$ & Notable pairwise comparisons $(p)$ \\
\hline P7/P8 & 2-UG: -0.82 & $F(2,28)=3.79$, & 2-UG, 3-UG(.001)* \\
& 3-UG: -1.43 & $p=.04, \eta_{p}{ }^{2}=0.21, \beta=0.64$ & \\
3-C: -1.34 & $F(2,26)=1.51$, & \\
PO7/PO8 & 2-UG: -0.80 & $p=.24, \eta_{p}^{2}=0.10, \beta=0.29$ & \\
& 3-UG: -1.27 & & 2-UG, 3-UG(.07) \\
TP7/TP8 & 3-C: -1.69 & $F(2,28)=4.33$, & 2-UG, 3-C(.09)
\end{tabular}

Note. Electrophysiological results from Experiment 2 at each electrode pair examined. The table columns indicate, from left to right, the electrode pair, the corresponding mean CDA amplitude values per condition, the statistical results, and notable pairwise comparisons. Asterisks next to the $p$ values in parentheses indicate a significant difference (alpha level: $p<.05)$.

maintenance period we parameterized the delay duration. The prediction was that connectedness might require a longer temporal processing window and that grouping-related benefits might emerge as delay period interval increased.

\section{Method}

\section{Participants}

Twenty new participants were tested in Experiment $2 \mathrm{~b}$ (15 female, mean age $=22.3$ years).
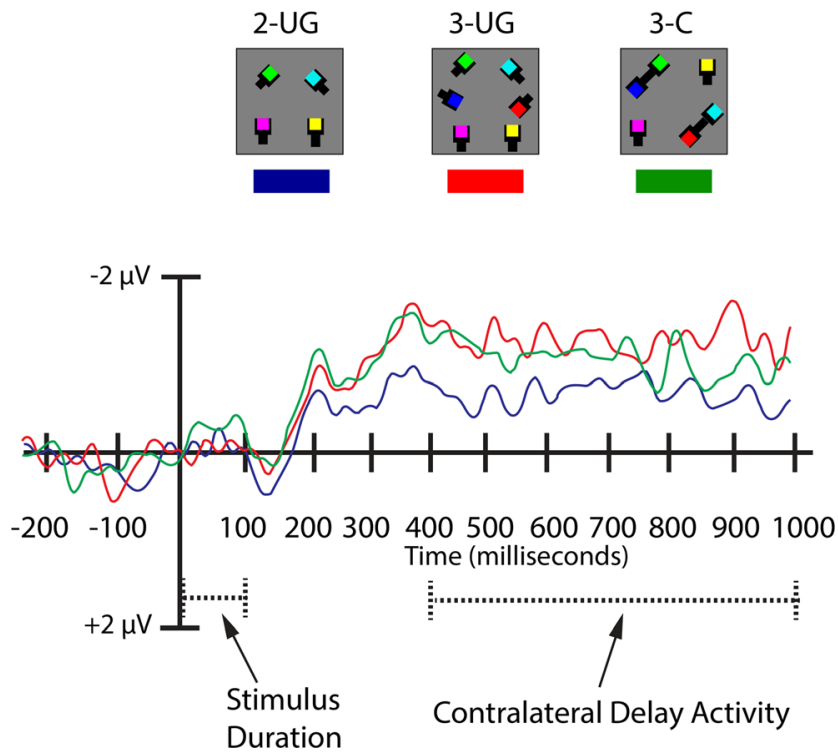

Fig. 4 Electrophysiological results for Experiment 2a. The timecourse of the CDA (in ms) is depicted along the abscissa. The amplitude scale (in microvolts) of the CDA for each experimental condition is indicated along the ordinate. The timecourse of the waveform depicts the prestimulus baseline period $(-200-0 \mathrm{~ms})$ occurring prior to the onset of the stimulus array for a given trial. The stimulus array is presented $(100 \mathrm{~ms}$, dashed line) and followed by a delay interval $(900 \mathrm{~ms})$. The measurement window for CDA analyses included amplitude values average across the delay period beginning at $400 \mathrm{~ms}$ and ending at $1,000 \mathrm{~ms}$ post stimulus onset (dashed line). The CDA amplitudes derived from electrode pair P7/P8 are depicted for each experimental condition.

\section{Procedure}

There were two changes from Experiment 2a. First, we did not record the EEG. Second, we used four delay periods (i.e., 600, $700,800$, and $900 \mathrm{~ms})$. Delay-period intervals were randomized such that there were an equal number of trials encompassing each delay period interval at each stimulus condition (24 trials per delay interval for each stimulus condition) for a total of 288 trials.

\section{Behavioral results}

We submitted the VWM accuracy data to a $3 \times 4$ repeated measures ANOVA including the factors of stimulus condition (2-UG, 3-UG, 3-C) and delay period $(600 \mathrm{~ms}, 700 \mathrm{~ms}, 800$ $\mathrm{ms}, 900 \mathrm{~ms}$ ); see Fig. 5. There was a main effect of stimulus condition, $F(2,38)=14.72, p<.001, \eta_{\mathrm{p}}^{2}=0.44, \beta=0.99$. Corrected pairwise comparisons revealed that this was driven by the expected higher accuracy in the 2-UG $(\mathrm{M}=0.89)$ condition compared to the 3-UG $(\mathrm{M}=0.83, \mathrm{p}=.007)$ and 3$\mathrm{C}$ conditions $(\mathrm{M}=0.81, \mathrm{p}=.001)$, replicating Experiment $2 \mathrm{a}$. There was no significant difference in accuracy between the 3$\mathrm{UG}$ and the $3-\mathrm{C}$ conditions $(p=.77)$. There was no main effect

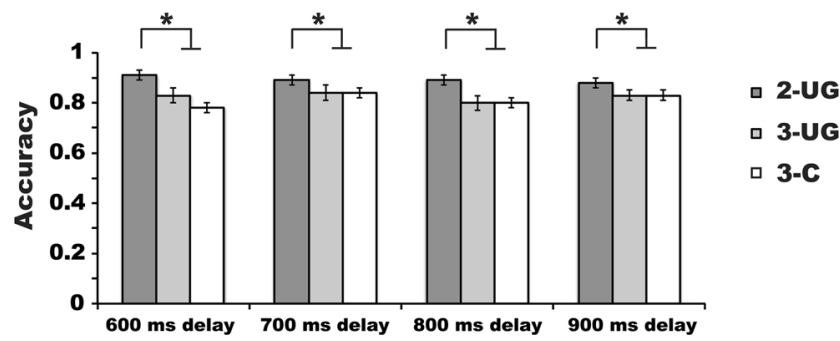

Fig. 5 Behavioral results from Experiment 2b. The abscissa depicts the delay period intervals used in the experiment. The legend shows the stimulus conditions. Accuracy is plotted along the ordinate by showing the proportion of correct trials. Asterisks and lines above the bars in the figure symbolize an observed significant difference between the two-item condition and each of the three-item conditions at each delay period interval; $\alpha=p<.05$. Error bars represent the standard errors of the means. 
of delay period, $\mathrm{F}(3,57)=1.30, \mathrm{p}=.28$, and no significant interaction between stimulus condition and delay period interval, $\mathrm{F}(6,114)=1.66, \mathrm{p}=.17$.

We also calculated accuracy in the 3-C condition as a function of probe type (grouped, ungrouped). A $2 \times 4$ repeated measures ANOVA with the factors of probe type (grouped, ungrouped) and delay period interval $(600,700,800$, or 900 $\mathrm{ms})$ showed no significant main effect of probe type, $F(3,57)=$ $2.42, p=.09$, or delay period, $F(3,57)=1.02, p=.33$. Importantly, there was a significant interaction between the factors of probe type and delay period interval, $F(3,57)=6.28, p=$ $.002, \eta_{p}^{2}=0.25, \beta=0.96$. Corrected pairwise comparisons revealed that this interaction was driven by a significant difference in accuracy between the grouped and ungrouped probe types for longer delay intervals ( $800 \mathrm{~ms}$ : grouped: $M=0.84$, ungrouped: $M=0.75, p=.04$; and $900 \mathrm{~ms}$ : grouped: $M=0.85$, ungrouped: $M=0.78, p=.05$ ). In contrast, at shorter delay periods there were trends in the opposite direction, indicating higher accuracy for the ungrouped probe items $(600 \mathrm{~ms}$ : grouped: $M=0.76$, ungrouped: $M=0.83, p=.06 ; 700 \mathrm{~ms}$ : grouped: $M=0.84$, ungrouped: $M=0.87, p=.48$ ).

The reaction time data showed no main effect of delay period interval, $F(3,57)=1.92, p=.14$. There was a main effect of condition, $F(2,38)=20.23, p<.001, \eta_{p}{ }^{2}=0.52, \beta=0.99$. Reaction times were shorter in the 2-UG $(M=956 \mathrm{~ms})$ relative to the 3-UG $(M=1,022 \mathrm{~ms}, p=.004$, and the 3-C conditions ( $M$ $=1,049 \mathrm{~ms}, p=.001$; there was no significant difference between the 3-UG and 3-C conditions ( $p=.13$ ). Finally, there was no significant interaction between delay period interval and stimulus condition, $F(6,114)=1.72, p=.12$.

\section{Discussion}

Experiments $2 \mathrm{a}$ and $2 \mathrm{~b}$ did not replicate previous reports demonstrating improved overall VWM performance with uniform connectedness (Woodman et al., 2003; Xu, 2006). We found no significant difference between the 3-UG and 3-C conditions (Experiments $2 \mathrm{a} / \mathrm{b}$ ). However, again there was evidence of a more nuanced benefit in probing a grouped item but only when the grouped items were probed later (i.e., 800, $900 \mathrm{~ms}$ ). There was actually a benefit to probing an ungrouped item earlier (i.e., 600, $700 \mathrm{~ms}$ ). This converges with the CDA (Experiment 2a) showing a selective late-delay reduction in 3-C CDA amplitude. The next question was to investigate the consequences of combining similarity and connectedness.

\section{Experiment 3: The influence of similarity of color and uniform connectedness}

Experiment 3 tested whether similarity and connectedness cues would interact and produce additional VWM benefits above what was observed in Experiments 1 and 2 when both were available either independently or together. We predicted that combining similarity and connectedness would improve VWM performance and reduce the amplitude of the CDA relative to three-item conditions containing one grouping cue, and potentially be superadditive. For instance, previous work in the perceptual domain has shown superadditive benefits of similarity and proximity when combined within the same stimulus array (e.g., Kubovy \& van den Berg, 2008). Alternatively, the conjunction of grouping cues would also provide an opportunity to observe interference between cue types within VWM.

\section{Method}

\section{Participants}

A new group of 25 adults participated in Experiment 3 (9 female, mean age $=27.12$ years). Six participants were excluded due to technical problems related to onset event marking during EEG data acquisition (1 participant), or severe artifact due to excessive eye movements during EEG recording (5 participants), leaving 19 participants.

\section{Stimuli and procedure}

Experiment 3 followed the protocol of Experiment 2a with the following exceptions. There were four conditions. The control condition was the 3-UG condition instead of the 2-UG condition. Second, we included the 3-SSP condition to test similarity/proximity benefits. The 3-C condition tested connectedness benefits. Finally, the conjunction of similarity/ proximity and connectedness, 3-SSP\&C, used matched colors in connected pairs; see Fig. 6 a.

\section{Results}

\section{Behavioral results}

Repeated-measures ANOVA on VWM accuracy revealed a significant main effect of stimulus condition, $F(3,54)=$ 23.86, $p<.001, \eta_{p}{ }^{2}=0.57, \beta=0.99$; see Fig. 6b. Corrected pairwise comparisons showed no benefit of uniform connectedness (3-UG: $M=0.81,3-\mathrm{C}: M=0.81, p=1.00$ ), but there was a significant penalty of similarity (3-SSP condition; $M=$ $0.74<3-\mathrm{UG}, 3-\mathrm{C} ; p \mathrm{~s}<.001)$, and a penalty associated with combined similarity and uniform connectedness (3-SSP\&C: $M=0.76<3$-UG, 3-C; both $p$ s $<.002$ ). There was no significant difference between the 3-SSP and the 3-SSP\&C conditions $(p=.24)$. The main effect of condition was observed for capacity (K) data, $F(3,54)=23.86, p<.001, \eta_{p}{ }^{2}=0.57, \beta=$ 0.99 , along with the same pattern of pairwise comparisons (all $p \mathrm{~s}<.01)$. 
a

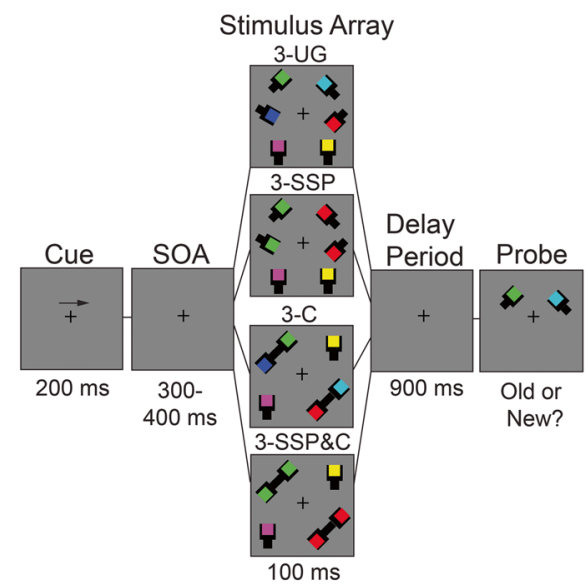

b

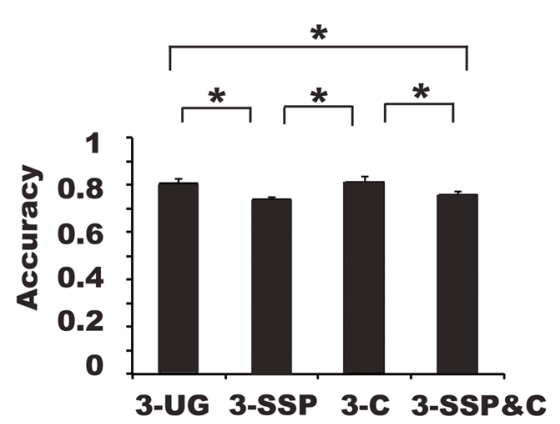

C

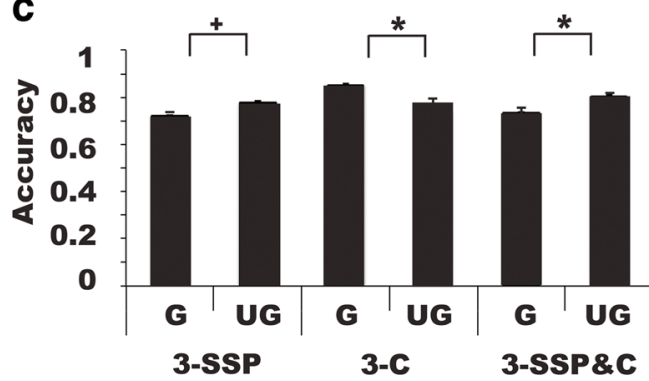

Fig. 6 Experiment 3 task paradigm, stimulus configurations, and behavioral results. A. Participants first viewed a fixation cross (300 $\mathrm{ms})$ and then were given an arrow cue $(200 \mathrm{~ms})$ indicating the side of the screen to which they should covertly attend during the trial. A variable interval $(300-400 \mathrm{~ms})$ preceded a memory array $(100 \mathrm{~ms})$ containing one of the four conditions displayed above: Three items (3-UG), three items in which two of the items were the same color (3-SSP), three items in which two of the items were connected (3-C), or three items in which two of the items were connected and shared the same color (3-SSP\&C). After a delay period $(900 \mathrm{~ms})$, a probed item appeared that was either the same color ("old" trials) as presented originally or a different color ("new" trials). Participants were given 3 seconds to respond. Note that stimuli are depicted for illustrative purposes only and do not reflect the exact dimensions and visual angles of the stimuli displayed during the actual experiment. B. VWM performance in Experiment 3. The abscissa depicts the conditions being compared. Accuracy (proportion correct) is plotted along the ordinate. C. Accuracy (proportion correct) as a function of probe type in the three grouped conditions is plotted along the ordinate. In panels $\mathrm{B}$ and $\mathrm{C}$, asterisks symbolize an observed significant difference between various conditions; $\alpha=p<.05$. In panel $\mathrm{C}$, the "+" sign indicates a borderline, nonsignificant effect $(p=.06)$. Error bars represent the standard error of the mean in each condition.
Next, a $2 \times 3$ repeated-measures ANOVA with the factors of probe type (grouped, ungrouped) and stimulus condition (3-SSP, 3-C, 3-SSP\&C) revealed a significant main effect of stimulus condition, $F(2,36)=10.22, p<.001, \eta_{p}{ }^{2}=0.36, \beta=$ 0.98 , that was driven by significantly higher accuracy in the 3 C condition $(M=0.81)$ compared to the 3 -SSP condition $(M=$ $0.74, p=.001)$; see Fig. $6 \mathrm{c}$. There was no main effect of probe type, $F(1,18)=0.04, p=.84$, but the interaction between probe type and stimulus condition reached significance, $F(2$, $36)=28.93, p<.001, \eta_{p}{ }^{2}=0.62, \beta=0.99$. In the $3-\mathrm{C}$ condition there was a significant benefit to probing a previously grouped $(M=0.86)$ rather than ungrouped item $(M=0.75$; $p=.001)$. In the 3-SSP\&C condition the pattern was the opposite; there was a benefit to probing a previously ungrouped item (ungrouped; $M=0.80$, grouped: $M=0.73, p=$ .03). A borderline effect was apparent in the 3-SSP condition (ungrouped: $M=0.77$; grouped: $M=0.71, p=.06$ ).

Repeated-measures ANOVA revealed a significant difference in reaction times as a function of stimulus condition, $F(3,54)=26.82, p<.001, \eta_{p}{ }^{2}=0.60, \beta=0.99$. Participants were significantly faster when responding to the 3-SSP trials $(M=806 \mathrm{~ms})$ compared to either the 3-UG trials $(M=862 \mathrm{~ms})$ or 3-C trials $(M=849 \mathrm{~ms}$; both $p \mathrm{~s}<.002)$. Moreover, participants were faster when responding to the 3SSP \&C trials $(M=795 \mathrm{~ms})$ compared to either the 3-UG trials or the 3-C trials (both $p$ s $<.002$ ). There was no significant difference between the 3-UG and 3-C conditions $(p=1.0)$ or between the 3-SSP and 3-SSP\&C conditions $(p=.49)$.

\section{Electrophysiological results}

Here we focused on electrode pair PO7/PO8; see Fig. 7, but see Table 3 for results for P7/P8 and TP7/TP8. A repeatedmeasures ANOVA comparing CDA amplitudes as a function of stimulus condition revealed a significant main effect, $F(3$, $54)=3.03, p=.05, \eta_{p}{ }^{2}=0.14, \beta=0.60$. This was driven by a significantly smaller CDA amplitude for the 3-SSP $(M=-0.68$ $\mu \mathrm{V})$ compared to the $3-\mathrm{C}$ condition $(M=-1.66 \mu \mathrm{V}, p=0.03)$. Apart from borderline nonsignificant differences between the 3-UG $(M=-0.99 \mu \mathrm{V})$ and 3-C $(p=0.08)$, and the 3-C and 3SSP \&C $(M=-0.88 \mu \mathrm{V}, p=0.09)$ conditions; no other differences emerged (all $p \mathrm{~s}>.25$ ).

As in Experiment 2a, we separately examined the early (400-600 ms) and late (800-1,000 ms) stages of the delayperiod activity recorded during Experiment 3 . The early delay period showed a main effect of condition, $F(3,54)=3.35$, $p=$ $.04, \eta_{p}{ }^{2}=0.16, \beta=0.65$. While none of the pairwise comparisons survived correction for multiple comparisons, the pattern of comparisons across conditions from the early delay period was similar to the overall analysis, which included the entire delay period. Specifically, the early mean CDA amplitude for 3-SSP $(-0.78 \mu \mathrm{V})$ was smaller than for 3-C (-1.85 $\mu \mathrm{V}, p=.15)$. Aside from this, no other differences in early 


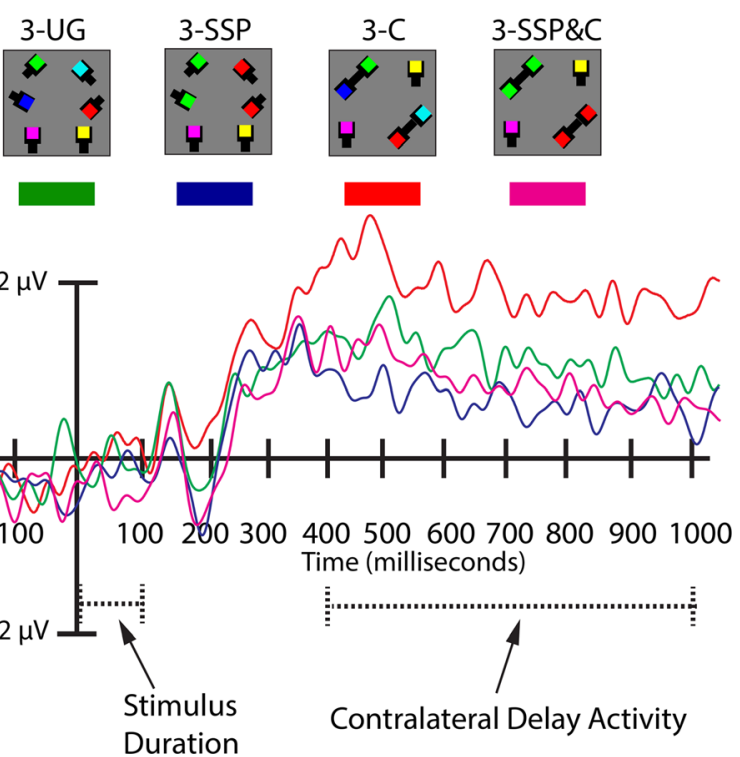

Fig. 7 Electrophysiological results for Experiment 3. The timecourse of the CDA (in ms) is depicted along the abscissa. The amplitude scale (in microvolts) of the CDA for each experimental condition is indicated along the ordinate. The timecourse of the waveform depicts the prestimulus baseline period $(-200-0 \mathrm{~ms})$ occurring prior to the onset of the stimulus array for a given trial. The stimulus array is presented (100 $\mathrm{ms}$, dashed line) and followed by a delay interval $(900 \mathrm{~ms})$. The measurement window for CDA analyses included amplitude values average across the delay period, beginning at $400 \mathrm{~ms}$ and ending at $1,000 \mathrm{~ms}$ post stimulus onset (dashed line). The CDA amplitudes derived from electrode pair PO7/PO8 are depicted for each experimental condition.

CDA amplitude emerged between these conditions or any remaining conditions $(3-\mathrm{UG}=-1.17 \mu \mathrm{V} ; 3-\mathrm{SSP} \& \mathrm{C}=-1.10$ $\mu \mathrm{V}$; all $p \mathrm{~s}>.42$ ).

A different pattern emerged during the late delay period (800-1,000 ms). Specifically, there was no significant main effect of stimulus condition with respect to mean CDA amplitude, $F(3,54)=2.25, p=.11, \eta_{p}{ }^{2}=0.11, \beta=0.54$. In fact, mean CDA amplitudes decreased for each condition from the early (i.e., $400-600 \mathrm{~ms})$ to late $(800-1,000 \mathrm{~ms})$ portion of the delay period (i.e., $3-\mathrm{UG}=-0.82 \mu \mathrm{V}$; 3-SSP $=-0.61 \mu \mathrm{V}$; 3-C $=$ $-1.54 \mu \mathrm{V} ; 3-\mathrm{SSP} \& \mathrm{C}=-0.68 \mu \mathrm{V})$.

\section{Discussion}

The results of Experiment 3 revealed interactions between similarity and connectedness with several different patterns of performance. First, there was evidence of a speedaccuracy tradeoff for similarity and similarity with connectedness - faster reaction times, accompanied by lower accuracy. Uniform connectedness significantly benefited VWM accuracy when a grouped item was probed. Moreover, the CDA data suggest that similarity and uniform connectedness reflect different underlying mechanisms. For instance, the amplitude associated with connectedness was largest, whereas the CDA amplitude associated with similarity was smallest. When both cues were available an intermediate CDA amplitude emerges. Were the influence of similarity due simply to a reduction in the overall number of colors/items to be maintained, we would have expected a reduction in the CDA for both the 3-SSP and 3-SSP\&C conditions. However, combining similarity and connectedness was not more of a good thing and interfered with VWM.

\section{General discussion}

Grouping cues benefit perception of visual arrays, and some evidence indicates these effects extend to VWM. Here, we tested whether we could replicate previously reported benefits to VWM provided by similarity, proximity, and uniform connectedness (Brady \& Tenenbaum, 2013; Gao et al., 2011; Peterson \& Berryhill, 2013; Morey et al., 2015; Shen et al.,

Table 3 Electrophysiological results per electrode pair from Experiment 3

\begin{tabular}{llll}
\hline Electrode pair & Mean amplitude per condition $(\mu \mathrm{V})$ & Analysis of variance & Notable pairwise comparisons $(p)$ \\
\hline P7/P8 & 3-UG: -1.04 & $F(3,54)=1.97$, \\
& 3-SSP: -0.88 & $p=.13, \eta_{p}^{2}=0.10, \beta=0.48$ & \\
& 3-C: -1.38 & & \\
3-SSP\&C: -0.65 & $F(3,54)=3.03$, & $3-\mathrm{UG}, 3-\mathrm{C}(.08)$ \\
PO7/PO8 & 3-UG: -0.99 & $p=.05, \eta_{p}^{2}=0.14, \beta=0.60$ & $3-\mathrm{SSP}, 3-\mathrm{C}(.03)^{*}$ \\
& 3-SSP: -0.68 & & \\
& 3-C: -1.66 & & \\
TP7/TP8 & 3-SSP\&C: -0.88 & & \\
& 3-UG: -0.84 & & \\
& 3-SSP: -0.44 & & \\
& 3-C: -1.04 & & \\
& 3-SSP\&C: -0.61 & & \\
\hline
\end{tabular}

Note. Electrophysiological results from Experiment 3 at each electrode pair examined. The table columns indicate, from left to right, the electrode pair, the corresponding mean CDA amplitude values per condition, the statistical results, and notable pairwise comparisons. Asterisks next to the $p$ values in parentheses indicate a significant difference (alpha level: $p<.05$ ). 
2013; Quinlan \& Cohen, 2012; Woodman et al., 2003; Xu, 2006; Xu \& Chun, 2007). We were interested in linking these VWM benefits with a neural signature, the CDA, to investigate their underlying basis. Our findings indicate that grouping-related VWM benefits can be accompanied by reduced neural demands, as indexed by the CDA. However, the data also suggest that all grouping cues are not the same, and they likely rely on different neural mechanisms. Given the extant perceptual configuration literature, it is surprising that we found limited benefits of connectedness in Experiments $2 \mathrm{a} / \mathrm{b}$ and 3 , although we did find a performance benefit when a previously grouped item was probed at test compared to ungrouped items. As such, while there is considerable overlap between perceptual and VWM processes, observing strong grouping effects in perception does not guarantee matching benefits in VWM. Importantly, the current experiments provide a cautionary tale regarding a priori assumptions that grouping benefits at the time of perception will lead to benefits when processing within VWM is required.

We replicated previously observed similarity benefits to VWM performance. We extended these findings by reporting a significantly reduced CDA (Experiment 1). Yet these benefits were erased when a second grouping cue, connectedness, was included in the experiment (Experiment 3). Connectedness was relatively less effective at improving VWM performance (Experiments 2a/b, 3). However, connectedness facilitated VWM performance when one of the connected items was probed. The CDA data from Experiment 2a suggested a slower emergence of connectedness-based VWM benefits. Experiment $2 b$ clarified that the VWM benefit associated with probing a grouped item emerged for longer delay period intervals (800, $900 \mathrm{~ms})$, suggesting slower processing of connectedness information in VWM than similarity. In Experiment 3, the availability of multiple grouping cues revealed interference rather than multiplying any VWM benefit. For instance, similarity (3-SSP) facilitated reaction times but hurt accuracy, as did similarity and connectedness together (3SSP\&C). The CDA amplitude was smallest for arrays containing similarity, intermediate for those with similarity and connectedness cues, and highest for those with connectedness.

Of particular relevance is an incomplete understanding of the effects of various grouping cues on perceptual performance (Wagemans et al., 2012). Additionally, the cortical mechanisms underlying perceptual grouping cues are only partially understood. One view is that top-down feedback between regions within visual cortex may instantiate grouping at the neural level, creating the perceptual experience of features or objects in the visual field "belonging together" (Murray, Schrater, \& Kersten, 2004). This view is consistent with the current findings that, with respect to VWM, topdown processes may underlie the beneficial effects of grouping cues on performance. Indeed, evidence of subtle benefits of connectedness as a function of probe type did not emerge until relatively late into the delay period (e.g., when using 800 and $900 \mathrm{~ms}$ delay-period intervals; Experiment 2b). Moreover, in the current study, the neural evidence of a connectedness benefit was a reduced CDA amplitude for VWM arrays containing connected items. However, this did not emerge in the context of the CDA until later in the maintenance period (e.g., Experiment 2a; $800-1,000 \mathrm{~ms})$. This is consistent with the possibility that grouping-related VWM benefits might take time to develop. This is consistent with recent findings that VWM binding processes develop throughout the maintenance period evident via a reduction in CDA amplitude, as intra-item features or separate items are integrated VWM (e.g., Luria \& Vogel, 2011). In the current work, the CDA amplitudes measured in response to the presence or absence of grouping cues in the memory array allowed for examination of the time course of these top-down processing influences across the duration of the maintenance period.

In contrast, including similarity improved VWM performance behaviorally and almost immediately reduced the CDA amplitude (e.g., Experiment 1). Unexpectedly, when similarity and connectedness were both available cues, interference was evident (e.g., Experiment 3). The interaction between these cues in VWM may relate to differences in cortical processing as has been proposed for perception (e.g., Sasaki, 2007). As such, distinct neural generators might correspond to the influence of similarity and connectedness cues in VWM. Given the coarse spatial resolution of ERP techniques, functional neuroimaging techniques will likely be required to dissociate the regions responsible for distinct grouping cues and between-cue interactions.

This work replicated previous reports of grouping-related benefits in VWM (Brady \& Tenenbaum, 2013; Gao et al., 2011; Lin \& Luck, 2009; Morey et al., 2015; Peterson \& Berryhill, 2013; Shen et al., 2013). We extended these findings by reporting a complementary pattern showing a smaller CDA amplitude for items grouped by similarity. Our use of more realistic grouping conditions with spatial variability extends recent work by Gao and colleagues (2011). They reported that VWM performance and CDA amplitude for four identical items was identical to the CDA for a single item. However, it is uncommon to use arrays composed of identical stimuli in VWM research. Additionally, the current experiments extend previous work showing biases toward processing previously grouped compared to ungrouped items (see also Peterson \& Berryhill, 2013; Woodman et al., 2003). VWM performance was generally better when the probed item was encoded as a member of a grouped pair. Importantly, this type of analysis can help reveal nuanced grouping benefits (e.g., those of connectedness revealed in Experiments 2a/b). 


\section{Theoretical implications and future research}

In the most relevant theoretical exploration of grouping cues, $\mathrm{Xu}$ considered three explanations for object-based VWM benefits related to connectedness (Xu, 2006). Xu suggested that (a) sharing a location (location/proximity hypothesis), (b) being physically connected (connectedness hypothesis), or (c) that both location and connectedness (hybrid hypothesis) might best characterize grouping benefits in VWM. The data from Xu (2006) supported a hybrid hypothesis because either increasing distance or reducing connectedness reduced grouping benefits and, consequently, both factors must contribute to the overall grouping benefit. The current data suggest that interactions between these features take time to develop and, for some grouping cues (e.g., similarity, proximity), may begin at VWM encoding and bias encoding success (Peterson \& Berryhill, 2013; Woodman et al., 2003). However, grouping-related benefits may also continue (e.g., similarity, proximity), or, in other cases (e.g., connectedness) do not even emerge until well into the VWM maintenance period. Evidence in favor of a maintenance contribution comes from fMRI and ERP findings. For instance, $\mathrm{Xu}$ and Chun (2007) and Xu (2008) found that arrays grouped via common region and connectedness, respectively, were associated with lower BOLD signal activation in the inferior intraparietal sulcus (IPS) relative to ungrouped arrays during the delay period of a VWM task. Additionally, similar to the findings of Anderson et al. (2013) and Gao et al. (2011), we observed a reduction in the CDA for grouped relative to ungrouped VWM arrays during VWM maintenance. The origin of these grouping effects on VWM processes warrants further investigation.

Other remaining questions include characterizing the full nature of these interactions and determining how other grouping cues may augment or detract from VWM performance. A second question would be to carefully probe the temporal dynamics of these interactions and their neural sources to clarify the underlying neural mechanisms of grouping related effects on VWM, as the current data support the view that distinct neural populations are engaged.

Perhaps the most exciting aspect of recently observed grouping-related benefits to VWM relates to the potential applications for VWM training paradigms. For instance, we did not explicitly refer to the presence of grouping cues within the stimulus arrays. However, alerting participants to the presence of grouping cues may lead to additional strategic, top-down facilitation of grouping-related benefits. Additionally, it may be the case that training strategies may enhance otherwise subtle grouping-related benefits.

\section{Conclusions}

These experiments expand our understanding of the processing of grouped stimuli in VWM. First, the results converge with previous findings from the VWM literature identifying the power of grouping principles to improve VWM performance (Anderson et al., 2013; Brady \& Tenenbaum, 2013; Gao et al., 2011; Lin \& Luck, 2009; Morey et al., 2015; Peterson \& Berryhill, 2013; Quinlan \& Cohen, 2012; Shen et al., 2013; Walker \& Davies, 2003; Woodman et al., 2003; Xu, 2006; Xu $\&$ Chun, 2007). Additionally, the results support previous findings that grouping-related behavioral benefits are accompanied by a reduction in the amplitude of the CDA, providing a neural index of the number of item representations being maintained within VWM (Gao et al., 2011). Second, the current results converge with previous work showing that the automaticity of perceptual organization to induce grouping between to-beremembered items is constrained by several factors previously shown to be important for efficient processing within VWM (e.g., initial object representation: Luria \& Vogel, 2014; stimulus configuration: Jiang et al., 2000; Jiang et al., 2004; encoding bias toward grouped items: Peterson \& Berryhill, 2013; Woodman et al., 2003).

The current findings highlight both the benefits and limitations associated with the presence of grouping cues within stimulus displays that require temporary storage in VWM. A variety of important visual stimuli in our immediate environment are only available for brief moments. Many of these stimuli must be maintained in VWM to meet the demands of cognitive tasks. Future VWM experiments examining these and other grouping cues will indicate whether imposing meaningful organization to our environment based on these principles could be beneficial to this important, but capacity limited, cognitive process.

Acknowledgements The authors declare no competing financial interests. Research reported in this manuscript was funded by NEI R15EY022775 to M. E. B and P20 GM103650 (P.I.: Michael Webster; Project Leader: M. E. B.). The content is solely the responsibility of the authors and does not necessarily represent the official views of the NIH, NIGMS or NEI.

\section{References}

Anderson, D. E., Vogel, E. K., \& Awh, E. (2011). Precision in visual working memory reaches a stable plateau when individual item limits are exceeded. Journal of Neuroscience, 31(3), 1128-1138.

Anderson, D. E., Vogel, E. K., \& Awh, E. (2013). Selection and storage of perceptual groups is constrained by a discrete resource in working memory. Journal of Experimental Psychology: Human Perception and Performance, 39(3), 824-835.

Baddeley, A. (2010). Working memory. Current Biology, 20(4), R136R140.

Bays, P. M., \& Husain, M. (2008). Dynamic shifts of limited working memory resources in human vision. Science, 321(5890), 851-854.

Berryhill, M. E., Peterson, D. J., Jones, K. J., \& Stephens, J. A. (2014). Hits and misses: Leveraging tDCS to advance cognitive research. Frontiers in Psychology, 5, 800.

Brady, T. F., \& Tenenbaum, J. B. (2013). A probabilistic model of visual working memory: Incorporating higher order regularities into 
working memory capacity estimates. Psychological Review, 120(1), 85-109.

Brainard, D. H. (1997). The Psychophysics Toolbox. Spatial Vision, 10(4), 433-436.

Cowan, N. (2001). The magical number 4 in short-term memory: A reconsideration of mental storage capacity. Behavioral Brain Science, 24(1), 87-114. discussion 114-185.

Duncan, J. (1984). Selective attention and the organization of visual information. Journal of Experimental Psychology: General, 113(4), 501-517.

Duncan, J., \& Humphreys, G. W. (1989). Visual search and stimulus similarity. Psychological Review, 96(3), 433-458.

Fukuda, K., Awh, E., \& Vogel, E. K. (2010). Discrete capacity limits in visual working memory. Current Opinion in Neurobiology, 20(2), $177-182$.

Gao, Z., Xu, X., Chen, Z., Yin, J., Shen, M., \& Shui, R. (2011). Contralateral delay activity tracks object identity information in visual short-term memory. Brain Research, 1406, 30-42.

Hollingworth, A. (2006). Visual memory for natural scenes: Evidence from change detection and visual search. Visual Cognition, 14(4/8), 781-807.

Jiang, Y., Chun, M. M., \& Olson, I. R. (2004). Perceptual grouping in change detection. Perception \& Psychophysics, 66(3), 446- 453.

Jiang, Y., Olson, I. R., \& Chun, M. M. (2000). Organization of visual short-term memory. Journal of Experimental Psychology: Learning, Memory, and Cognition, 26(3), 683-702.

Kahneman, D., \& Henik, A. (1977). Effects of visual grouping on immediate recall and selective attention (Vol. 6). Hillsdale, NJ: Erlbaum.

Kahneman, D., \& Treisman, A. (1984). Changing views of attention and automaticity. New York, NY: Academic Press.

Kristjánsson, (2006). Surface assignment modulates object-formation for visual short-term memory. Perception, 35, 865-881.

Kubovy, M., \& van den Berg, M. (2008). The whole is equal to the sum of its parts: A probabilistic model of grouping by proximity and similarity in regular patterns. Psychological Review, 115, 131-154.

Lin, P. H., \& Luck, S. J. (2009). The influence of similarity on visual working memory representations. Visual Cognition, 17(3), 356372.

Luck, S. J., \& Vogel, E. K. (1997). The capacity of visual working memory for features and conjunctions. Nature, 390(6657), 279-281.

Luck, S. J., \& Vogel, E. K. (2013). Visual working memory capacity: From psychophysics and neurobiology to individual differences. Trends in Cognitive Sciences, 17(8), 391-400.

Luria, R., \& Vogel, E. K. (2011). Shape and color conjunction stimuli are represented as bound objects in visual working memory. Neuropsychologia, 49(6), 1632-1639.

Luria, R., \& Vogel, E. K. (2014). Come together, right now: Dynamic overwriting of an object's history through common fate. Journal of Cognitive Neuroscience, 26, 1819-1826.

Mack, A., \& Rock, I. (1998). Inattentional blindness. Cambridge, MA: MIT Press.

Mack, A., Tang, B., Tuma, R., Kahn, S., \& Rock, I. (1992). Perceptual organization and attention. Cognitive Psychology, 24, 475-501.

Moore, C. M., \& Egeth, H. (1997). Perception without attention: evidence of grouping under conditions of inattention. Journal of Experimental Psychology: Human Perception and Performance, 23(2), 339-352.

Morey, C. C., Cong, Y., Zheng, Y., Price, M., \& Morey, R. D. (2015). The color-sharing bonus: Roles of perceptual organization and attentive processes in visual working memory. Archives of Scientific Psychology, 3, 18-29.
Murray, S. O., Schrater, P., \& Kersten, D. (2004). Perceptual grouping and the interactions between visual cortical areas. Neural Networks, $17,695-705$.

Neisser, U. (1967). Cognitive psychology. New York, NY: AppletonCentury-Crofts.

Palmer, S., \& Rock, I. (1994). Rethinking perceptual organization: The role of uniform connectedness. Psychonomic Bulletin \& Review, 1(1), 29-55.

Pashler, H. (1988). Familiarity and visual change detection. Perception \& Psychophysics, 44, 369-378.

Pelli, D. G. (1997). The VideoToolbox software for visual psychophysics: Transforming numbers into movies. Spatial Vision, 10(4), 437-442.

Peterson, D. J., \& Berryhill, M. E. (2013). The Gestalt principle of similarity benefits visual working memory. Psychonomic Bulletin \& Review, 20(6), 1282-1289.

Quinlan, P. T., \& Cohen, D. J. (2012). Grouping and binding in visual short-term memory. Journal of Experimental Psychology: Learning, Memory, and Cognition, 38(5), 1432-1438.

Sasaki, Y. (2007). Processing local signals into global patterns. Current Opinion in Neurobiology, 17(2), 132-139.

Shen, M., Yu, W., Xu, X., \& Gao, Z. (2013). Building blocks of visual working memory: Objects or Boolean maps? Journal of Cognitive Neuroscience, 25(5), 743-753.

Simons, D. J., \& Levin, D. T. (1997). Change blindness. Trends in Cognitive Science, 1(7), 261-267.

Simons, D. J., \& Rensink, R. A. (2005). Change blindness: Past, present, and future. Trends in Cognitive Science, 9(1), 16-20.

Todd, J. J., \& Marois, R. (2004). Capacity limit of visual short-term memory in human posterior parietal cortex. Nature, 428(6984), $751-754$.

Vogel, E. K., \& Machizawa, M. G. (2004). Neural activity predicts individual differences in visual working memory capacity. Nature, 428(6984), 748-751.

Wagemans, J., Elder, J. H., Kubovy, M., Palmer, S. E., Peterson, M. A., Singh, M., \& von der Heydt, R. (2012). A century of Gestalt psychology in visual perception: I. Perceptual grouping and figureground organization. Psychonomic Bulletin \& Review, 138(6), $1172-1217$.

Walker, P., \& Davies, S. J. (2003). Perceptual completion and objectbased representations in short-term visual memory. Memory \& Cognition, 31(5), 746-760.

Watson, S. E., \& Kramer, A. F. (1999). Object-based visual selective attention and perceptual organization. Perception \& Psychophysics, 61(1), 31-49.

Wertheimer, M. (1950). Gestalt theory. New York, NY: Humanities Press.

Woodman, G. F., Vecera, S. P., \& Luck, S. J. (2003). Perceptual organization influences visual working memory. Psychonomic Bulletin \& Review, 10(1), 80-87.

$\mathrm{Xu}$, Y. (2006). Understanding the object benefit in visual short-term memory: The roles of feature proximity and connectedness. Perception \& Psychophysics, 68(5), 815-828.

$\mathrm{Xu}$, Y. (2008). Representing connected and disconnected shapes in human inferior intra-parietal sulcus. NeuroImage, 40, 1849-1856.

Xu, Y., \& Chun, M. M. (2006). Dissociable neural mechanisms supporting visual short-term memory for objects. Nature, 440(7080), 91-95.

$\mathrm{Xu}$, Y. D., \& Chun, M. M. (2007). Visual grouping in human parietal cortex. Proceedings of the National Academy of Sciences of the United States of America, 104(47), 18766-18771. 